\section{AORTIC VALVE REPLACEMENT WITH FREEHAND AUTOLOGOUS PERICARDIUM}

Fifty-one patients with a mean age of 31.2 years underwent aortic valve replacement with glutaraldehyde-treated autologous pericardium. Pure aortic regurgitation was present in $28(54.9 \%)$, stenosis in 9 , and mixed disease in 14. Simultaneous mitral valve repair was done in 17 patients and replacement in 1. There were no hospital and two late deaths. Three patients required reoperation because of failure of the pericardial valve as a result of infective endocarditis in two (5 and 31 months after operation) and commissural tear at 8 months in another. One patient underwent reoperation at 24 months because of failure of the mitral valve repair. The pericardial aortic valve, which had $2+$ regurgitation since the first operation, was also replaced. Macroscopic and microscopic examination findings in the excised pericardium were excellent. No thromboembolic events have been detected and no patient received anticoagulation therapy except one after mitral valve reoperation and replacement with a mechanical valve. The actuarial survival was $84.53 \% \pm 12.29 \%$ at 60 months, freedom from failure of the aortic reconstruction $83.83 \% \pm 8.59 \%$, and freedom from any event $72.59 \% \pm 12.79 \%$. Doppler echocardiographic study at most recent follow-up showed a mean gradient of $12.56 \pm 8.10 \mathrm{~mm} \mathrm{Hg}$ and mean regurgitation on a scale from 0 to $4+$ of $0.80 \pm 0.66$. Although the maximum follow-up is only 5 years, the results obtained so far encourage us to continue replacing the aortic valve with stentless autologous pericardium. (J Thorac Cardiovasc SuRg 1995;110:511-6)

Carlos M. G. Duran, MD, PhD, Begonia Gometza, MD, Naresh Kumar, MD, Ricardo Gallo, MD, and Rafael Martin-Duran, MD, Riyadh, Saudi Arabia
A utologous pericardium, because of its ready availability, ease of handling, and low cost, has been used regularly since the early days of cardiac surgery. However, because of intermittent reports of its tendency to retract or become aneurysmal, the general opinion has been negative. Our positive experience with triple aortic cusp extension with bovine pericardium ${ }^{1}$ and the description by Chachques and associates ${ }^{2}$ of a rapid method to

From the Department of Cardiovascular Diseases, King Faisal Specialist Hospital and Research Center, Riyadh, Saudi Arabia.

Supported in part by external grant AT-11-12 from the King Abdulaziz City for Science and Technology, Riyadh, Saudi Arabia.

Received for publication July 27, 1994.

Accepted for publication Jan. 26, 1995.

Address for reprints: Carlos M. G. Duran, MD, Chairman, Department of Cardiovascular Diseases, King Faisal Specialist Hospital and Research Center, P.O. Box 3354, Riyadh, Saudi Arabia

Copyright 우 1995 by Mosby-Year Book, Inc.

$0022-5223 / 95 \$ 3.00+0 \quad \mathbf{1 2 / 1 / 6 3 6 6 8}$ stabilize pericardium encouraged us to initiate a series of total aortic valve replacement with glutaraldehyde-treated autologous pericardium. This paper reports our total experience in 51 patients.

\section{Patients and methods}

Beginning in April 1989, 51 consecutive patients underwent aortic valve replacement with autologous pericardium. Patient selection was based on the preferences of the individual surgeon and does not represent a truly general population because initially only young patients with expected problems of permanent anticoagulation were selected. With experience and given the positive findings in terms of low transvalvular gradients in patients with a small aortic anulus, the indications were expanded to include older patients with calcific stenosis.

The preoperative patient data are shown in Table I. The mean age was 31.2 years with a range of 14 to 68 and a median of 26 years. Fifteen patients were female and 36 male. The cause of disease was rheumatic in $43(84.3 \%)$, degenerative in $5(9.8 \%)$, congenital in 2 , and infective in 1. The mean preoperative New York Heart Association (NYHA) functional class was 2.57. Forty-seven (93\%) patients were in sinus rhythm and four in atrial fibrillation. The diagnosis was established with transthoracic color Doppler echocardiography in all cases. Coronary angiog- 
Table I. Preoperative data in 51 patients with aortic valve replacement with autologous pericardium

\begin{tabular}{|c|c|c|c|c|c|c|c|c|c|}
\hline \multirow[b]{2}{*}{ Diagnosis } & \multirow[b]{2}{*}{$n(\%)$} & \multirow{2}{*}{$\begin{array}{c}\text { Mean } \\
\text { age } \\
(y r)\end{array}$} & \multirow[b]{2}{*}{$\begin{array}{l}\text { Rheumatic } \\
\text { cause (\%) }\end{array}$} & \multirow{2}{*}{$\begin{array}{l}\text { Sinus } \\
\text { rhythm } \\
(\%)\end{array}$} & \multirow{2}{*}{$\begin{array}{c}\text { Mean } \\
\text { NYHA } \\
\text { class }\end{array}$} & \multirow[b]{2}{*}{$\begin{array}{c}\text { Mean } A R \text { (on scale } \\
\text { from } 1-4+\text { ) }\end{array}$} & \multirow{2}{*}{$\begin{array}{c}\text { Mean } \\
\text { gradient } \\
\text { (mm Hg) }\end{array}$} & \multicolumn{2}{|c|}{$\begin{array}{c}\text { Associated mitral } \\
\text { valve disease }\end{array}$} \\
\hline & & & & & & & & $n$ & $\begin{array}{l}\text { Percent having } \\
\text { repair }\end{array}$ \\
\hline AR & $28(54.9)$ & 24 & $27(96.4)$ & $26(92.9)$ & 2.43 & $3.52 \pm 0.54$ & $11.12 \pm 4.44$ & 11 & 100 \\
\hline AS & $9(17.6)$ & 53.2 & $5(55.0)$ & $7(77.8)$ & 2.89 & $0.75 \pm 0.38$ & $53 \pm 10.57$ & 2 & 100 \\
\hline Mixed & $14(27.4)$ & 31.5 & $11(78.6)$ & $14(100)$ & 2.64 & $2.69 \pm 0.72$ & $39.42 \pm 11.45$ & 5 & 80 \\
\hline Total & $51(100)$ & 31.2 & $43(84.3)$ & $47(93)^{\prime}$ & 2.57 & $2.78 \pm 1.15$ & $29.48 \pm 20.80$ & 18 & 94.4 \\
\hline
\end{tabular}

$A R$, Aortic valve regurgitation; $A S$, aortic valve stenosis; Mixed, aortic valve stenosis and regurgitation.

raphy was done only in male patients older than 45 years and female patients older than 50 years unless the clinical data suggested that it was necessary. No patient in this series had coronary artery disease. Twenty-eight $(54.9 \%)$ patients had pure aortic regurgitation, 9 aortic stenosis, and 14 mixed lesions. Mitral valve disease was present in 18 patients and tricuspid valve disease in 1 . All patients underwent intraoperative transesophageal echocardiography (TEE). Specific and essential data searched for were the diameter of the aortic anulus, as measured from opposing cusp insertion points in the long axis view, and the presence of a trileaflet valve. Calcification, even of the anulus was no contraindication.

Myocardial protection was achieved, initially, with cold crystalloid potassium cardioplegia and, since 1991, with antegrade and retrograde cold blood cardioplegia. The heart was vented in every case. If there was involvement of other valves, they were exposed first and, in the case of the mitral valve, treated appropriately first. In general, mitral valve replacement was considered a contraindication to aortic valve reconstruction.

The whole surgical technique of valve reconstruction was an attempt at reproducing as closely as possible the normal aortic leaflets tailored to the individual patient. In accordance with the work of Swanson and Clark, ${ }^{3}$ who provided the measures of the aortic valve relative to the aortic anulus, plastic containers with three consecutive bulges of different sizes were made. These bulges reproduced the three valve leaflets with measures corresponding to the different aortic valve diameters. After the midline sternotomy was completed, a rectangular strip of pericardium was resected with a total length of about $1 \mathrm{~cm}$ more than three times the TEE-measured aortic anulus diameter and a width of one diameter. After it was cleaned, the pericardium was placed in the appropriate container filled with $0.5 \%$ buffered glutaraldehyde. The pericardial strip was held in position, over the three bulges, with a perforated plastic sheath of the same dimension as the negative of the bulges. After fixation for 10 minutes the pericardium was rinsed for another 10 minutes and trimmed down. The pericardium was sutured to the aortic valve remnant or to the anulus with a single 4-0 polypropylene running suture following a technique described in detail elsewhere. ${ }^{4}$ Basically, suturing started at the midpoint of each sinus and was stopped against the suture of the next cusp. The portion of the pericardial strip between leaflets was sutured as a new commissure to the aortic wall. The two extremities of the strip were similarly sutured to the aortic wall as the third new commissure (Fig. 1). After the patient was weaned from bypass TEE was done in all cases.

All patients were followed up in a dedicated valve clinic by two physicians 8 weeks and 6 months after operation and at yearly intervals thereafter. If a patient failed to attend the clinic he or she was contacted by a social worker who had visited them during their hospital stay. The preoperative, surgical, postoperative, and follow-up data were entered into a software program (PATS, Dendrite, Portland, Ore.). All events were noted according to the guidelines issued by The American Association for Thoracic Surgery and the Society of Thoracic Surgeons. ${ }^{5}$ Statistical significance of the echocardiographic data was analyzed with the Student's $t$ test.

\section{Results}

Between April 1989 and June 1994, 51 patients underwent aortic valve replacement with glutaraldehyde-treated autologous pericardium. Eighteen patients had simultaneous mitral valve operation with 17 repairs and 1 replacement with a bioprosthesis. The mean cardiopulmonary bypass time for the whole group of patients was 131.8 minutes and the crossclamp time 96.9 minutes. For those patients with an isolated aortic valve operation the mean bypass time was 122.8 minutes and the mean crossclamp time 87.9 minutes.

There were no hospital deaths although in four patients not included in this series the result of the reconstruction was not considered satisfactory and a replacement with a prosthesis was undertaken without mortality. Three patients were lost to follow-up (94.1\% complete) with a maximum of 61 months and mean of 21.2 months. The total follow-up was 88.97 patient-years. There were no thromboembolic events. Two late deaths (3.9\%) have occurred: one patient died in a car accident and the second died in another hospital apparently of left ventricular failure with a competent aortic valve. There were three reoperations, without mortality, which were necessary because of failure of the reconstruction $(5.8 \%)$. The causes were endocarditis in two patients ( 5 and 31 months after operation) and tear of a cusp near 


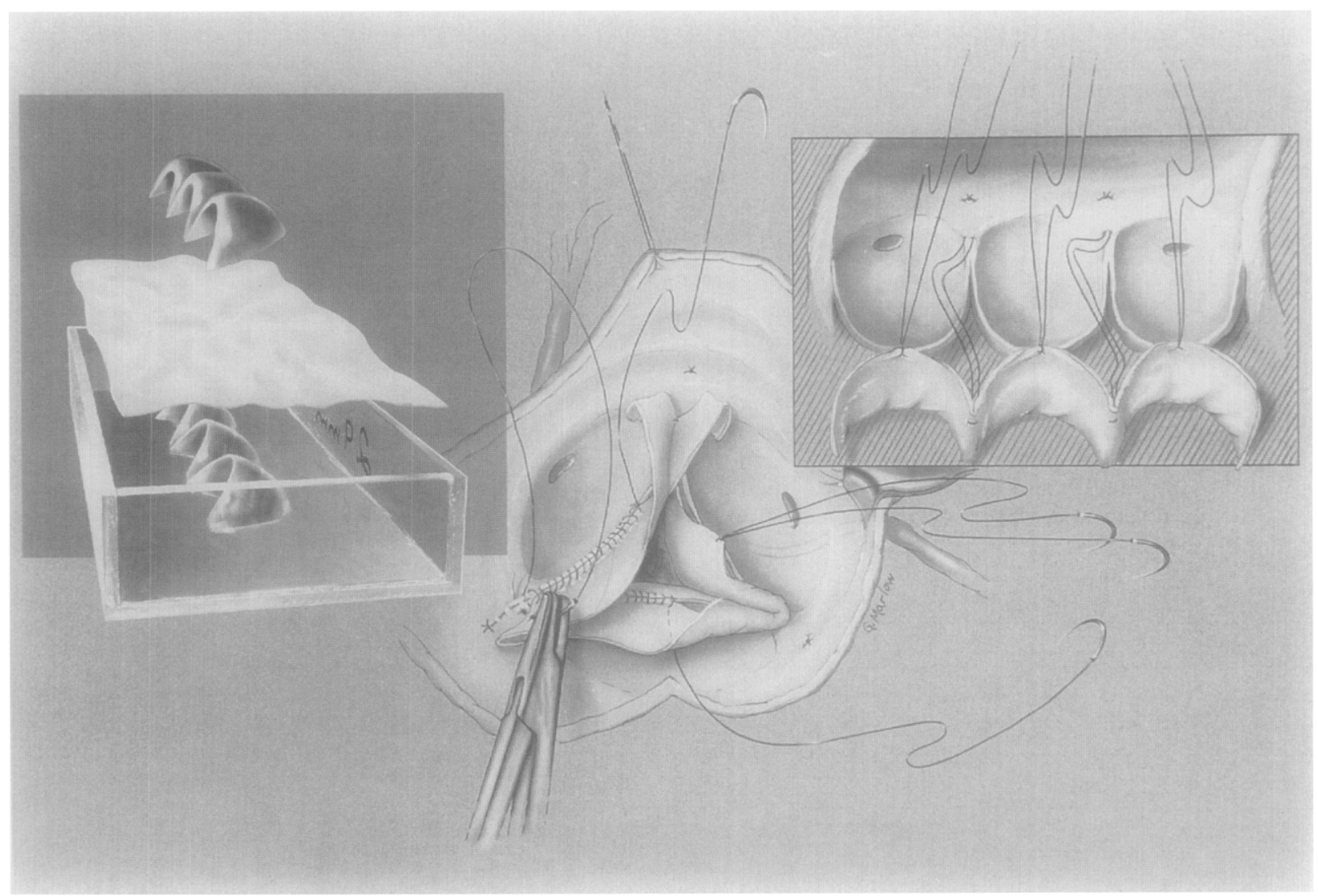

Fig. 1. Surgical technique for aortic valve replacement with autologous pericardium. Left inset, Tanning of autologous pericardium. Right inset, Anchoring sutures.

one commissure in the third patient 8 months after operation. One patient required reoperation because of failure of the mitral valve repair 24 months after the initial operation. The aortic valve in this patient, which had a $2+$ regurgitation score since. the first operation, was replaced because the dysfunctioning mitral valve was replaced with a mechanical valve and the possibility of a third operation was rejected by the patient. The autologous pericardium was perfectly healed, thin, and mobile without any thrombus or macroscopic fibrin deposition or calcification (Fig. 2). Histologic examination showed perfect conservation of the collagen fibers, no cell infiltration, and patchy neointima. At the base of the cusps on the arterial aspect a thin fibrous and cellular layer could be observed that extended for a few millimeters (Fig. 3).

The mean postoperative NYHA functional class at most recent visit was 1.22 . The actuarial survival was $84.53 \% \pm 12.29 \%$ at 60 months. Freedom from failure of the reconstruction was $83.83 \% \pm 8.59 \%$ and the actuarial freedom from any event was $72.59 \% \pm$ $12.79 \%$ again at 60 months after operation (Fig. 4).
The echocardiographic data of the patients before operation, at discharge from the hospital, and at most recent follow-up are shown in Table II. Noteworthy findings were the range of aortic anulus diameters measured intraoperatively, which varied from 17 to $31 \mathrm{~mm}$, and the significant difference in degree of aortic regurgitation between the preoperative and postoperative measurements. There was no significant change between the early and late postoperative grade of regurgitation and transvalvular gradients, which advocates the stability of the technique at medium range. The mean postoperative gradients were low, which is particularly important in the stenotic lesions.

\section{Discussion}

Aortic valve cusp extension with pericardium has a long history dating to 1963 when $\operatorname{Ross}^{6}$ reported one case of aortic insufficiency treated with single cusp enlargement with autologous fresh pericardium. In 1964 Björk and Hultquist ${ }^{7}$ decribed the pathologic findings of two cases of cusp extension with presumably autologous fresh pericardium: one 


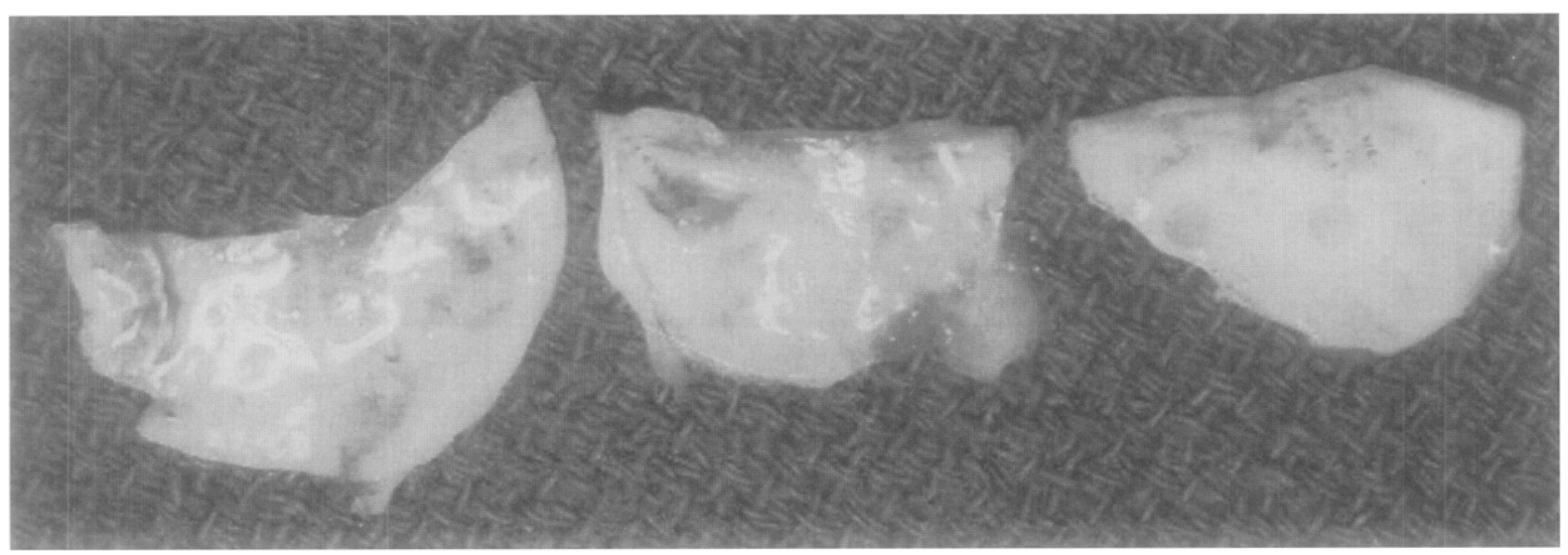

Fig. 2. Macroscopic appearance of autologous pericardium explanted 2 years after implantation. Reoperation was necessary because of mitral valve dysfunction.

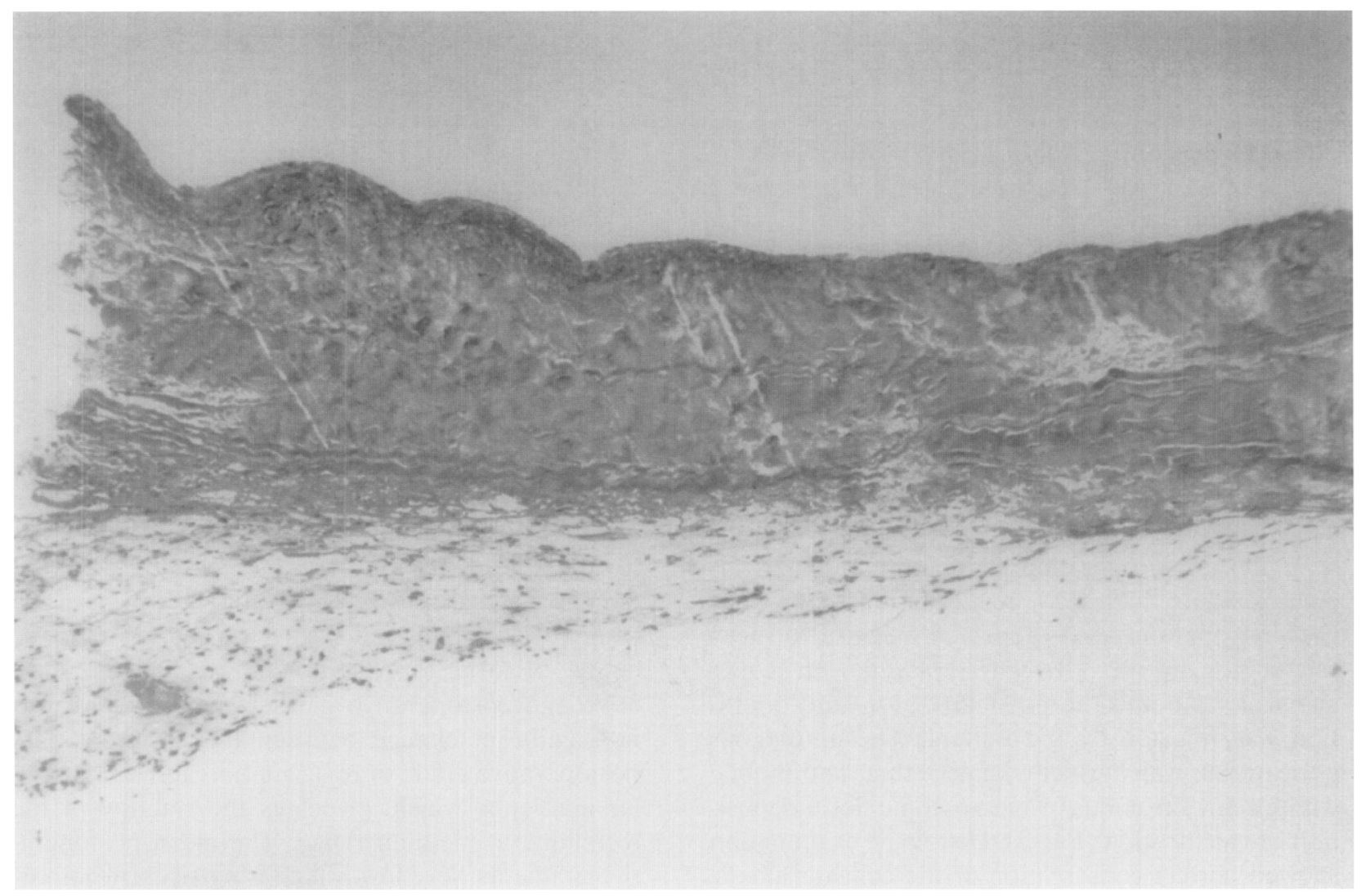

Fig. 3. Cross section of autologous pericardium 2 years after implantation. Lower aspect corresponds to aortic surface (Masson's trichrome stain; original magnification $\times 50$ ).

patient died 5 months after operation with a thickened and calcified pericardium and the other at reoperation for severe valvular insufficiency present since the first operation done $3 \frac{1}{2}$ months previously. Edwards ${ }^{8}$ in 1969 described a surgical technique for total valve replacement with autogenous tissue studied in vitro and applied with pericardium in two patients: one of the patients died 1 week later with a competent valve at autopsy. Bahnson and associates $^{9}$ in 1970 reported two cases of single leaflet 


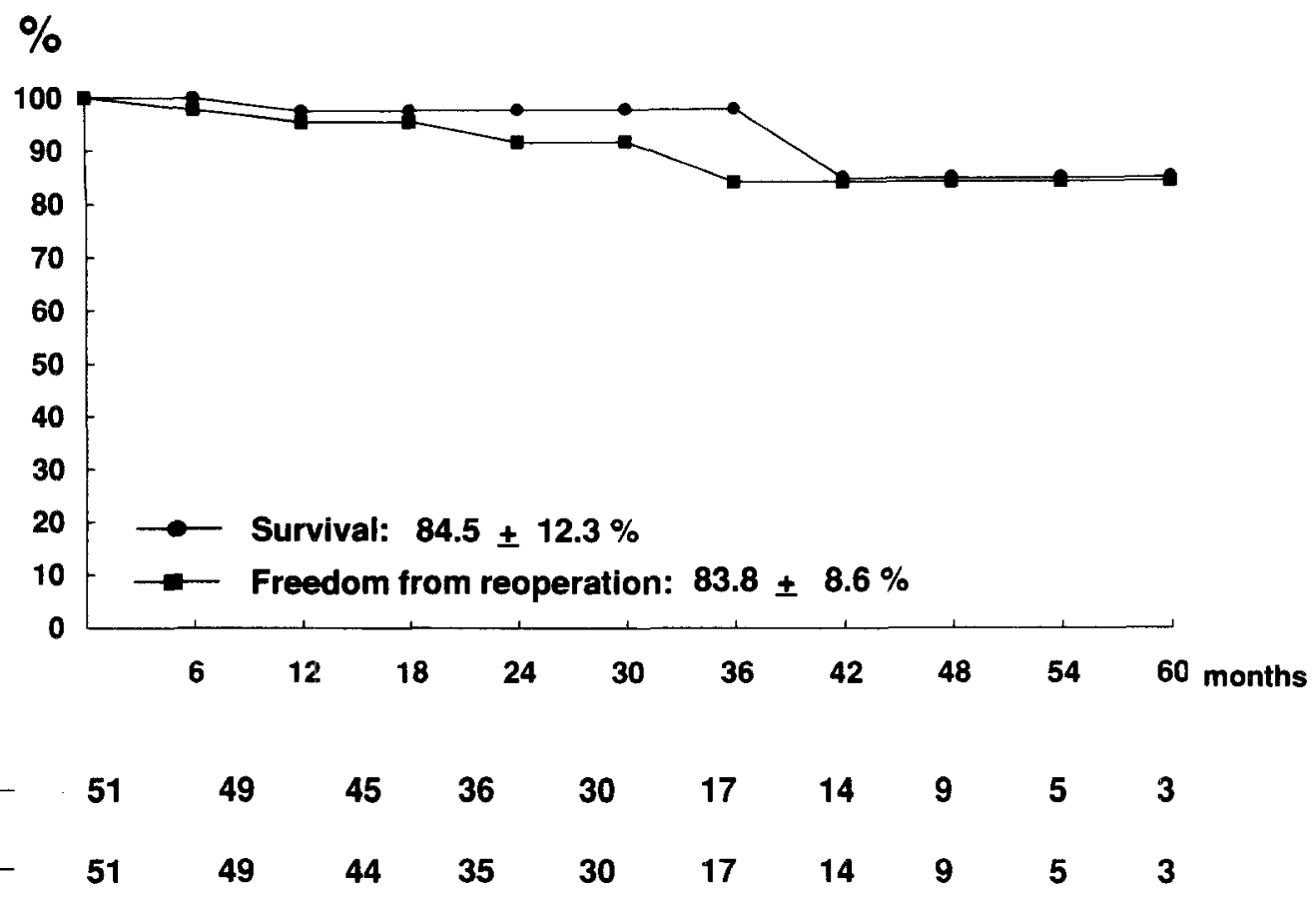

Fig. 4. Actuarial survival and freedom from reoperation curves in 51 patients with autologous pericardium aortic valve replacement.

Table II. Color Doppler echocardiographic data in 51 patients with autologous pericardium aortic valve replacement

\begin{tabular}{ccccc}
\hline & $A R$ & $A S$ & Mixed & Total \\
\hline Anulus diameter (mm) & $27.33 \pm 2.09$ & $20.20 \pm 2.59$ & $21.88 \pm 4.05$ & $24.52 \pm 4.10$ \\
$\quad$ (range) & $(24-31)$ & $(18-23)$ & $(17-30)$ & $(17-31)$ \\
Preoperative findings & & & & \\
$\quad$ AR & $3.52 \pm 0.54^{*}$ & $0.75 \pm 0.38$ & $2.69 \pm 0.72^{*}$ & $2.78 \pm 1.15^{*}$ \\
$\quad$ Gradient (mm Hg) & $11.12 \pm 4.44$ & $53 \pm 10.57^{*}$ & $39.42 \pm 11.45^{*}$ & $29.48 \pm 20.80^{*}$ \\
Discharge findings & & & & \\
AR & $0.69 \pm 0.53$ & $0.69 \pm 0.46$ & $0.75 \pm 0.40$ & $0.69 \pm 0.48$ \\
$\quad$ Gradient (mm Hg) & $9.18 \pm 3.65$ & $16.43 \pm 11.16$ & $8.83 \pm 2.62$ & $10.21 \pm 5.87$ \\
Most recent follow-up & & & & \\
AR & $0.82 \pm 0.78$ & $0.75 \pm 0.38$ & $0.81 \pm 0.60$ & $0.80 \pm 0.66$ \\
Gradient (mm Hg) & $11.94 \pm 7.07$ & $16.57 \pm 13.72$ & $10.91 \pm 3.73$ & $12.56 \pm 8.10$ \\
\hline
\end{tabular}

Mean grade of aortic regurgitation as determined on scale from 0 to $4+$. Gradient is the Doppler mean transvalvular gradient in millimeters of mercury. $A R$, Aortic regurgitation; $A S$, aortic stenosis; Mixed, aortic stenosis and regurgitation.

${ }^{*} p<0.001$ significance versus discharge and most recent follow-up.

replacement and three cases of triple leaflet extensions with autologous pericardium. Although all patients had residual regurgitation, three of them were doing well $1 \frac{1}{2}, 41 / 2$, and $4 \frac{1}{2}$ years after operation. These isolated and inconclusive attempts remained practically forgotten and displaced by the reliability of the new prostheses. The availability of glutaraldehyde-treated xenogeneic pericardium encouraged Yacoub and associates ${ }^{10}$ to use calf pericardium treated in $0.2 \%$ glutaraldehyde for 7 days in
45 patients with an actuarial survival of $89 \%$ at 7 years. Batista and associates ${ }^{11}$ in 1986 reported excellent results with a single strip of glutaraldehyde-treated bovine pericardium in 60 consecutive patients. The same group later reported no calcifications in 216 patients followed up for up to 6 years. ${ }^{12}$ Because of the youth and difficulty of permanent anticoagulation in our patients, it was decided to follow the technique already described by Batista and associates ${ }^{11}$ in which as little as possible 
of the cusp free edge was resected and a large strip of commercially available glutaraldhyde-treated bovine pericardium was sutured to the three cusps together with the construction of very high new commissures. This technique was applied beginning in July 1988 to 27 patients with excellent results to date in all patients, except in one who at 58 months was seen with calcification of one cusp. The intraoperative observation in four cases of severe electrocardiographic ischemic changes that were transient in three but that necessitated valve replacement in the fourth alerted us to a possible pitfall of this technique. The report of four early deaths caused by "myocardial dysfunction" in the series of Batista ${ }^{11}$ in very young patients and the direct visual observation of the reconstructed valve through a cystoscope ${ }^{13}$ which showed bending of the free edge of the pericardium toward the sinuses of Valsalva, confirmed the suspicion. A new surgical technique for the total replacement of the aortic valve was developed in the experimental laboratory in which autologous pericardium was shaped into three cusps during the glutaraldehyde fixation.

Two main questions must be addressed when the use of this approach is being considered. The first is the need for a standard surgical technique that ensures a correct, reproducible, and safe result in terms of immediate competence and low transvalvular gradients. The second is the long-term durability of the selected material. The absence of hospital mortality and transient or permanent ischemic changes and the findings of echocardiographic valve competence and nonsignificant gradients are indicators of a reliable surgical technique. In terms of durability, the maximum follow-up of 61 months is still too short. Two patients had to undergo reoperation because of endocarditis. This nearly $5 \%$ prevalence is a cause of concern particularly when the main problem in the experience of Senning ${ }^{14}$ with fresh autologous fascia lata was infective endocarditis present in $14 \%$ of his cases. In our experience, the different anatomic localization and the pretreatment of the pericardium with glutaraldehyde should reduce this potential danger. One patient had to undergo reoperation because of rupture of the left coronary leaflet next to the right and left commissure. This area corresponds to where the two ends of the strip of pericardium must be joined when the new commissure is fashioned and this area might have been either damaged or insufficiently supported. The excellent macroscopic and microscopic findings in the explanted pericardium 2 years after implantation are encouraging. It is believed that being stentless and nonantigenic this tissue should perform at least better than the standard bioprosthesis.

The immediate and late echocardiographic data are also encouraging not only in terms of valve competence but also as regards gradients, even in the presence of a small aortic anulus. This last finding has allowed an expansion of the indications for this procedure to those older patients with small stenotic and calcified aortic valves.

\section{REFERENCES}

1. Duran CMG, Kumar N, Gometza B, Al Halees Z. Indications and limitations of aortic valve reconstruction. Ann Thorac Surg 1991;52:447-54.

2. Chachques JL, Vasseur B, Perrier P, et al. A rapid method to stabilize biological materials for cardiovascular surgery. Ann N Y Acad Sci 1988;529:184-6.

3. Swanson M, Clark RE. Dimensions and geometric relationships of the human aortic valve as a function of pressure. Circ Res 1974;35:871-82.

4. Duran CMG, Gallo R, Kumar N. Aortic valve replacement with autologous pericardium: surgical technique. J Card Surg 1995;10:1-9.

5. Edmunds LH, Clark R, Cohn LH, et al. Guidelines for reporting morbidity and mortality after cardiac valvular operations. J THoraC CARdiovasC SURG 1988;96:351-3.

6. Ross DN. Surgical reconstruction of the aortic valve. Lancet 1963;1:571-4.

7. Björk VO, Hultquist G. Teflon and pericardial aortic valve prosthesis. J Thorac CARDIOvasC SURG 1964; 47:693-702.

8. Edwards WS. Aortic valve replacement with autogenous tissue. Ann Thorac Surg 1969;8:126-32.

9. Bahnson HT, Hardesty RL, Baker LD, Brookes D II, Gall DA. Fabrication and evaluation of tissue leaflets for aortic and mitral valve replacement. Ann Surg 1970;171:939-47.

10. Yacoub M, Khaghani A, Dhalla N, et al. Aortic valve replacement using unstented dura or calf pericardium: early and medium term results. In: Bodnar E, Yacoub M, eds. Biological and bioprosthetic valves. New York: Yorke Medical Books, 1986:684-90.

11. Batista RJV, Dobrianskij A, Comazzi M, et al. Clinical experience with stentless pericardial aortic monopatch for aortic valve replacement. J THORAC CARDIOVASC SURG 1987;93:19-26.

12. Batista RJV. In discussion of David TE, Pollick C, Boss J. Aortic valve replacement with stentless pocine aortic bioprosthesis. J Thorac CARDIovasc SuRg 1990;99:113-8.

13. Ghandour M, Al Halees Z, Duran C. A simple method for intraoperative visualization of the repaired aortic valve. J Thorac Cardiovasc SuRg 1994;107:632-3.

14. Senning A. Fascia lata replacement of aortic valves. $\mathbf{J}$ Thorac Cardiovasc Surg 1967;54:465-70. 